\title{
Vegetarian diets and risks of total and site-specific fractures: results from the prospective EPIC-Oxford study
}

\author{
Tammy Y.N. Tong, Paul N. Appleby, Aurora Perez-Cornago and Timothy J. Key \\ Cancer Epidemiology Unit, Nuffield Department of Population Health, University of Oxford, Richard Doll Building, \\ Old Road Campus, Oxford, United Kingdom
}

\section{Abstract}

Introduction: It has been speculated that vegetarians or vegans may have higher risks of fractures than meat eaters, but there is limited evidence from prospective cohorts. We aimed to assess the risks of total and site-specific fractures in people of different diet groups, in a prospective cohort with a large proportion of non-meat eaters.

Materials and methods: In EPIC-Oxford, dietary information was collected at baseline (1993-2001) and at follow-up around 14 years later $(\approx 2010)$. Participants were categorised into five diet groups $(\approx 20,106$ regular meat eaters: $\geq 50 \mathrm{~g}$ of meat per day, $\approx 9,274$ low meat eaters: $<50 \mathrm{~g}$ of meat per day, $\approx 8,037$ fish eaters, $\approx 15,499$ vegetarians and $\approx 1,982$ vegans, with minor variations in numbers for each outcome after pre-specified exclusions) at both time points. Using multivariable Cox regression adjusted for socio-demographic, lifestyle, and physiological confounders, we estimated the risks of total and site-specific fractures (arm, wrist, hip, leg, ankle, and other main sites i.e. clavicle, rib and vertebra) in the different diet groups, with outcomes identified through record linkage.

Results: Over an average of 17.6 years of follow-up, we observed 3,941 cases of total fractures, 566 arm fractures, 889 wrist fractures, 945 hip fractures, 366 leg fractures, 520 ankle fractures, and 467 other main site fractures. Compared with meat eaters, vegetarians had marginally higher risks of total fractures (hazard ratios and 95\% confidence intervals: $1.10 ; 1.00-1.20)$ and arm fractures $(1.28$; 1.01-1.63), while vegans had significantly higher risks of total fractures $(1.44 ; 1.21-1.72)$ and leg fractures $(2.06$; $1.22-3.47)$, and marginally higher risks of arm fractures $(1.60,1.01-2.54)$. For hip fractures, the risks were higher in fish eaters (1.28; 1.03-1.59), vegetarians $(1.27 ; 1.05-1.55)$ and vegans $(2.35 ; 1.67-3.30$, p-heterogeneity $<0.0001)$ than regular meat eaters. There were no significant differences in risks of wrist, ankle or other main site fractures by diet groups. Overall, the significant associations appeared stronger without adjustment for body mass index (e.g. 1.52; 1.27-1.81 in vegans for total fractures), and were slightly attenuated with additional adjustment for total protein $(1.41 ; 1.17-1.69)$ or dietary calcium $(1.32 ; 1.10-1.59)$.

Discussion: In conclusion, non-meat eaters, especially vegans, had higher risks of either total or some site-specific fractures, particularly hip fractures. The higher risks might be partly explained by the lower body mass index in these diet groups, but differences in dietary intakes of protein and calcium are likely relevant as well. Given the observational design of this study, causality and potential mechanisms should be further investigated.

\section{Conflict of Interest}

There is no conflict of interest. 